\title{
Prospective clinical validation of a meningioma consistency grading scheme: association with surgical outcomes and extent of tumor resection
}

\author{
Kyohei Itamura, BS, Ki-Eun Chang, MD, Joshua Lucas, MD, Daniel A. Donoho, MD, \\ Steven Giannotta, MD, and Gabriel Zada, MD, MS \\ Department of Neurological Surgery, University of Southern California, Los Angeles, California
}

OBJECTIVE The present study aims to assess the clinical utility of a previously validated intraoperative meningioma consistency grading scale and its association with extent of resection (EOR) and various surgical outcomes.

METHODS The previously validated grading system was prospectively assessed in 127 consecutive patients undergoing open craniotomy for meningioma by multiple neurosurgeons at two high-volume academic hospitals from 2013 to 2016. Consistency grading scores ranging from 1 (soft) to 5 (firm/calcified) were retrospectively analyzed to test for association with surgical outcomes and EOR, categorized as gross-total resection (GTR) or subtotal resection, defined by postoperative MRI.

RESULTS One hundred twenty-seven patients were included in the analysis with a tumor consistency distribution as follows: grade 1,3.1\%; grade 2, 14.2\%; grade 3, 44.1\%; grade 4,32.3\%; and grade 5, $6.3 \%$. The mean tumor diameter was $3.6 \pm 1.7 \mathrm{~cm}$. Tumor consistency grades were grouped into soft (grades 1 and 2), average (grade 3), and firm (grades 4 and 5) groups for statistical analysis with distributions of $17.3 \%, 44.1 \%$, and $38.6 \%$, respectively. There was no association between meningioma consistency and maximal tumor diameter, or location. Mean duration of surgery was longer for tumors with higher consistency: grades 1 and 2, 186 minutes; grade 3, 219 minutes; and grades 4 and 5, 299 minutes $(p=0.000028)$. There was a trend toward higher perioperative complication rates for tumors of increased consistency: grades 1 and 2, 4.5\%; grade 3,7.0\%; and grades 4 and $5,20.8 \%$ ( $p=0.047)$. The proportion of GTR for each consistency group was as follows: grades 1 and 2, 77\%; grade 3,68\%; and grades 4 and $5,43 \%(p=0.0062)$.

CONCLUSIONS In addition to other important meningioma characteristics such as invasiveness, tumor consistency is a key determinant of surgical outcomes, including operative duration and EOR. Future studies predicting tumor consistency based on preoperative neuroimaging will help considerably with preoperative planning for meningiomas.

https://thejns.org/doi/abs/10.3171/2018.7.JNS1838

KEYWORDS consistency; extent of resection; meningioma; clinical validation; grading scale; oncology

$\mathrm{M}$ ENINGIOMAS are the most common primary intracranial tumors in adults ${ }^{12}$ and may arise from any part of the nervous system where arachnoid cap cells are present. ${ }^{7}$ As such, they are often located in areas in which surgical access is limited, such as the skull base. Recent advancement in endoscopy has afforded a wider and more comprehensive view of the surgical corridor, and growing interest in tailored keyhole craniotomies has allowed similar degrees of resection through more cosmetically appealing incisions. ${ }^{4,5,11,22}$ However, safe utilization of these approaches requires a thorough preoperative understanding of the surgical anatomy and tumor characteristics.

One such characteristic of skull base tumors that affects surgical difficulty and extent of resection (EOR) is tumor consistency. ${ }^{14}$ However, a standardized description of meningioma consistency has not been established due to a lack of objective and comprehensive grading criteria. Although several variations of classification systems have been proposed, most are limited by subjective binary de- 
TABLE 1. Meningioma consistency grading system

\begin{tabular}{|c|c|c|c|}
\hline $\begin{array}{l}\text { Consistency } \\
\text { Score }\end{array}$ & General Description & Capsule Characteristics & $\begin{array}{c}\text { Exemplary Instrument(s) Used for Internal } \\
\text { Debulking }\end{array}$ \\
\hline 1 & Extremely soft tumor & $\begin{array}{l}\text { Capsule easily folded or no } \\
\text { capsule }\end{array}$ & Suction \\
\hline 2 & $\begin{array}{l}\text { Soft tumor; bulk of tumor freely suctioned; fibrous } \\
\text { stroma remains }\end{array}$ & $\begin{array}{l}\text { Capsule easily folded following } \\
\text { partial internal debulking }\end{array}$ & $\begin{array}{l}\text { Majority done w/ suction; fibrous stroma resected } \\
\text { w/ capsule }\end{array}$ \\
\hline 3 & $\begin{array}{l}\text { Average consistency; cannot be freely suctioned; } \\
\text { requires some degree of mechanical debulking }\end{array}$ & $\begin{array}{l}\text { Capsule folds following suf- } \\
\text { ficient debulking }\end{array}$ & $\begin{array}{l}\text { Piecemeal resection; ultrasonic aspiration or } \\
\text { other mechanical debulking device, often at } \\
\text { low setting; sharp dissection }\end{array}$ \\
\hline 4 & Firm tumor; requires mechanical debulking & $\begin{array}{l}\text { Firm capsule; difficult to } \\
\text { collapse despite tumor } \\
\text { debulking }\end{array}$ & $\begin{array}{l}\text { Piecemeal resection, ultrasonic aspiration, loop } \\
\text { cautery, or other mechanical debulking, often } \\
\text { at high setting; sharp resection or loop cautery }\end{array}$ \\
\hline 5 & $\begin{array}{l}\text { Extremely firm \&/or calcified tumor; may approach } \\
\text { density of bone; often requires en bloc resection }\end{array}$ & $\begin{array}{l}\text { Rigid capsule that does not } \\
\text { fold or collapse }\end{array}$ & $\begin{array}{l}\text { Difficult to debulk even w/ ultrasonic aspiration, } \\
\text { cautery loop, or mechanical/sharp dissection }\end{array}$ \\
\hline
\end{tabular}

From Zada et al: Neurosurg Focus 35(6):E1, 2013. Published with permission.

scriptions such as "soft" and "hard." 21 Furthermore, none of these proposed criteria have been rigorously tested for statistical and clinical validity, and are often obtained from retrospective chart reviews of operative reports. As such, there is a clear need for a reproducible grading system that can be used to reliably communicate the consistency of meningiomas among neurosurgeons, neuroradiologists, and neuropathologists.

Our group previously proposed and validated a 5-point grading scale for intraoperative consistency of meningiomas based on the surgeon's ability to debulk and collapse the tumor capsule. ${ }^{23}$ The study reported high interuser reliability and laid the groundwork for further investigation into its clinical application. The present study aims to assess this scale in a clinical context and test the hypothesis that tumor consistency is associated with EOR during open craniotomy for meningioma resection.

\section{Methods}

\section{Consistency Grading}

A 5-point grading scale was previously developed and validated by Zada et al. ${ }^{23}$ to characterize the intraoperative consistency of meningiomas (Table 1). The authors designed a practical and objective scale based on the surgeon's ease and ability to internally debulk the tumor and collapse the capsule of the meningioma. Grade 1 meningiomas are soft, liquefied tumors amenable to internal debulking with suction alone. Grade 2 meningiomas are also relatively soft tumors that can be partially debulked with suctioning; however, there are interspersing fibrous stroma that require additional resection, along with an easily collapsible capsule. Grade 3 meningiomas are those with average consistency that cannot be freely suctioned and require some mechanical debulking (e.g., ultrasonic aspiration, piecemeal debulking, or a cutting-aspiration device), with a capsule that is easily collapsed following internal debulking. Grade 4 meningiomas are firm tumors for which internal debulking is a challenge, requiring sharp resection, loop cautery, or ultrasonic aspiration at a higher setting; in grade 4 tumors, it is a challenge to fold the fibrous tumor capsule despite internal debulking, and often requires extracapsular dissection. Finally, grade 5 meningiomas are extremely fibrous or calcified lesions, with a consistency approaching bone, and internal debulking and capsule folding is typically unsuccessful.

\section{Study Design}

Meningioma consistency scores were prospectively assigned at the time of surgery for 127 consecutive patients undergoing open craniotomy for resection of intracranial meningiomas at Keck Hospital of the University of Southern California (USC) or Los Angeles County + USC Medical Center from 2013 to 2016. Consistency scores were recorded on a separate sheet of paper immediately following the operation, and dictated into the operative note after each case. The basic tumor histology and grade for all histologically verified meningiomas were available for most of the tumors in the final pathology report, but were not included in this current analysis. Maximal tumor diameter was determined on preoperative contrast-enhanced T1weighted MRI.

A retrospective chart review was conducted for all patients to whom a consistency grade was prospectively assigned. IRB approval was obtained in accordance with our institution's standards. For the purposes of this study, patients with smaller tumors that were resected in an en bloc fashion (without requiring internal debulking) were excluded. Extent of resection in our study was defined on the basis of the postoperative contrast-enhanced T1-weighted MRI, interpreted by an independent neuroradiologist. Gross-total resection (GTR) was defined as no remaining enhancing portion of the tumor that was defined on preoperative imaging.

\section{Statistical Analysis}

For statistical purposes, consistency scores were grouped into 3 groups: soft (grades 1 and 2), average (grade 3), and firm (grades 4 and 5). Statistical significance was 


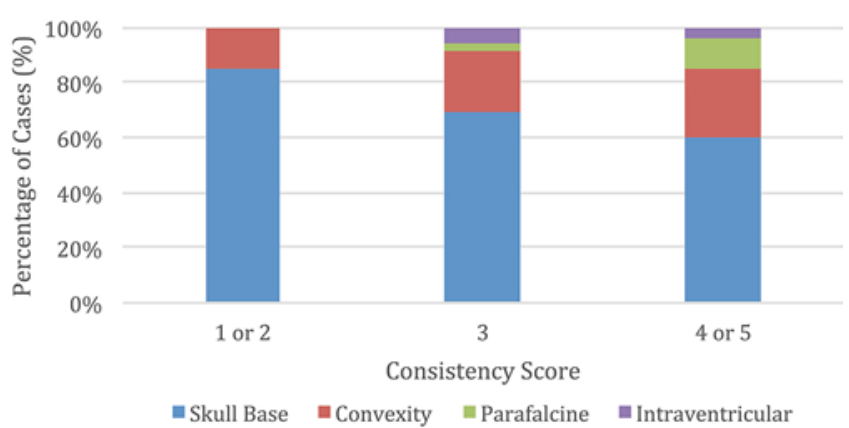

FIG. 1. Graphic representation of the correlation between tumor location and consistency score. Figure is available in color online only.

defined as $\mathrm{p}<0.05$. All statistical analyses were performed using SPSS (IBM Corp.). Consistency scores were subjected to chi-square analysis for independence with EOR and perioperative complications. A 1-way ANOVA was used to determine the relationship between consistency and maximal diameter, length of surgery, and duration of hospital stay.

\section{Results}

\section{Patient Demographics and Tumor Characteristics}

One hundred twenty-seven patients were included in the analysis. The mean $( \pm \mathrm{SD})$ patient age was $56 \pm 11$ years, and there were 80 women (63.0\%) and 47 men (37.0\%).

The mean maximal tumor diameter $( \pm \mathrm{SD})$ was 3.6 $\pm 1.7 \mathrm{~cm}$. The distribution of overall tumor consistency scores was as follows: grade 1,3.1\%; grade $2,14.2 \%$; grade $3,44.1 \%$; grade $4,32.3 \%$; and grade $5,6.3 \%$. For statistical analysis, individual grades were grouped as follows: soft (grades 1 and 2,17.3\%), average (grade 3,44.1\%), and firm (grades 4 and 5,38.6\%).

Tumor location for each case was categorized as skull base, convexity, parafalcine, and intraventricular. The highest frequency of tumors was found on the skull base, followed by the convexity, and then the parafalcine (Fig. 1). There was no statistically significant difference between tumor location and EOR, or consistency.

Mean maximal tumor diameter as determined by preoperative MRI for each category was as follows: grades 1 and 2,3.4 $\pm 1.1 \mathrm{~cm}$; grade $3,3.9 \pm 1.7 \mathrm{~cm}$; and grades 4 and $5,3.5 \pm 1.2 \mathrm{~cm}$. A 1-way ANOVA was performed to determine the effect of tumor consistency on tumor size. The ANOVA showed that the effect of consistency on tumor size was not significant: $F(2,124)=1.46, p=0.24$.

\section{Relationship Between Consistency Score and Surgical Outcomes}

To further elucidate the clinical application of the proposed consistency grading system, we further analyzed the relationship between consistency scores and duration of hospital stay, duration of surgery, and perioperative complications.

The mean duration of surgery for each category was as follows: grades 1 and 2, $186 \pm 35$ minutes; grade 3, $219 \pm$ 50 minutes; and grades 4 and 5, 299 \pm 55 minutes. A 1-way ANOVA was performed to determine the effect of tumor consistency on duration of surgery. The ANOVA showed
TABLE 2. List of perioperative complications of soft (1 or 2), average (3), and firm (4 or 5 ) tumor resection

\begin{tabular}{ccc}
\hline $\begin{array}{c}\text { Consistency } \\
\text { Score }\end{array}$ & $\begin{array}{c}\text { Total } \\
\text { Complications } \\
(\%)\end{array}$ & \multicolumn{1}{c}{ Description } \\
\hline 1 or 2 & $1(4.5)$ & Cranial nerve palsy \\
\hline 3 & $4(7.0)$ & $\begin{array}{c}\text { Venous infarct, transient arm weak- } \\
\text { ness, PICA stroke, transient aphasia }\end{array}$ \\
\hline 4 or 5 & $10(20.8)$ & $\begin{array}{c}\text { Carotid injury, cranial nerve palsy } \times 2, \\
\text { hemiparesis } \times 3, \text { transient CSF leak, } \\
\text { aphasia, delayed chronic hematoma, } \\
\text { frontal lobe syndrome }\end{array}$ \\
\hline
\end{tabular}

PICA = posterior inferior cerebellar artery.

that the effect of consistency on duration of surgery was significant: $\mathrm{F}(2,124)=51.9, \mathrm{p}=0.000028$.

The mean duration of hospital stay for each category was as follows: grades 1 and 2, $6.5 \pm 2.0$ days; grade 3, $7.2 \pm 2.7$ days; and grades 4 and 5, $7.4 \pm 3.5$ days. A 1-way ANOVA was performed to determine the effect of tumor consistency on duration of hospital stay. The ANOVA showed that the effect of consistency on hospital duration was not significant: $\mathrm{F}(2,124)=0.84, \mathrm{p}=0.43$.

The perioperative complication rate for each category was as follows: grades 1 and 2, 4.5\%; grade 3, 7.0\%; and grades 4 and 5,20.8\%. Complications after surgery included any episodes of cranial nerve palsy, hemiparesis, venous infarcts, transient extremity weakness, stroke, and visual loss within the postoperative hospital course (Table 2). A chi-square test of independence was performed to determine the association between consistency scores and number of complications. Categorical grouping was maintained during analysis. The relation between these variables was statistically significant $\left(\chi^{2}=6.12, p=0.047\right)$.

\section{Relationship Between Consistency Score and EOR}

The proportion of GTR for each category was as follows: grades 1 and 2,77\%; grade 3,68\%; and grades 4 and $5,43 \%$. A chi-square test of independence was performed to determine the association between consistency scores and EOR. Categorical grouping was maintained during analysis. The relation between these variables was statistically significant $\left(\chi^{2}=10.2, p=0.0062\right.$; Fig. 2$)$.

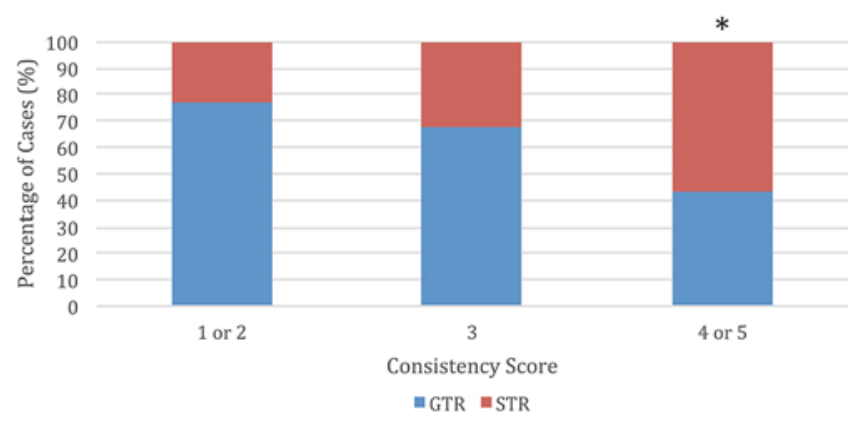

FIG. 2. Graphic representation of the correlation between extent of resection (GTR/subtotal resection [STR]) and consistency score. ${ }^{*} p<$ 0.05 . Figure is available in color online only. 


\section{Discussion}

Reliable preoperative evaluation of tumor consistency is of paramount importance for meningioma resection, as it helps with surgical planning and tailoring the most optimal surgical approach that is likely to facilitate maximal safe tumor resection. Our retrospective analysis of prospectively assigned tumor consistency scoring shows that EOR is associated with meningioma consistency.

Over the past few decades the inherent property of tumor consistency has become an important variable in meningioma surgery, and a priori knowledge of tumor consistency holds significant value in predicting surgical outcome and planning a surgical approach. Minimally invasive strategies may be preferentially selected when there is reasonable confidence that the lesion will lend itself to safe resection via the proposed exposure.

The grading system proposed by our group in 2013 provides a practical and easy-to-use method with high interuser reliability for the objective assessment of tumor consistency in intracranial meningiomas. ${ }^{23}$ It was created in response to the existing variability and subjectivity of prior studies that have attempted to characterize, predict, and convey meningioma consistency. Our grading system is unique, as it is based on an ordinal scale rather than simply labeling meningiomas as either "soft" or "hard." The impetus for our approach also derived from the common practice in neuroimaging studies of retrospectively using this binary approach based on neurosurgical operative reports, a method that fails to recognize areas of mixed consistency within the tumor. Using the numerical scale described above, two independent surgeons evaluated 50 consecutive meningiomas in a prospective fashion and found a high degree of user agreement for overall tumor consistency. ${ }^{23}$ Using a numerical grading scheme that encompasses the entire consistency characteristic of the tumor as a whole allows for more objective comparison of studies examining meningioma consistency. ${ }^{10}$

The purpose of the present study was to demonstrate the clinical validity of our proposed consistency scale with respect to EOR. Our results showed a statistically significant increase in the rate of GTR associated with softer meningiomas. We also found that the duration of surgery was significantly longer for firmer lesions (grades 4 and 5) and complication rates were increased with increasing consistency, although this did not meet statistical significance.

These findings are consistent with previous studies that have shown the importance of tumor consistency on surgical outcome and overall survival. ${ }^{6,15,16}$ Our numerical grading scale provides an objective and validated scheme for effective communication of the intraoperative tumor consistency. Ultimately, future studies correlating preoperative imaging characteristics to our consistency scale can help design the best operative strategy to maximize the degree of resection and afford the best chance of a surgical cure.

There are numerous prior studies evaluating meningioma consistencies with conventional and novel MRI techniques. Hyperintensity on T2-weighted imaging has been found to correlate with softer tumors with higher water content. In contrast, low signal intensity on T2weighted imaging has been related to harder tumors due to increased collagen and calcium content ${ }^{1,10,15,17}$ Although
T1-weighted imaging itself has not been correlated with tumor consistency, ${ }^{1,17,18,20}$ meningiomas that were hyperintense on T2-weighted imaging and hypointense on T1-weighted imaging were more likely to be soft, while those that were hypointense on T2-weighted imaging and isointense on T1-weighted imaging were more likely to be firm. ${ }^{2}$ Furthermore, there is considerable variability as to whether FLAIR and/or proton density-weighted imaging are of any help in determining tumor consistency.13,18 Similarly, the handful of studies examining the utility of diffusion-weighted imaging in predicting tumor consistency have found confounding results. ${ }^{2}$ MR elastrography is an emerging advanced MRI technique that is meant to provide a measure of tissue stiffness similar to manual palpation. ${ }^{9}$ Stiffness is determined by evaluating shear wave movement through tissues. ${ }^{8}$ Although its application for intracranial pathology is limited, there are few recent reports that correlate increased stiffness to intratumoral consistency. ${ }^{3,19}$

\section{Limitations and Future Directions}

Our study is not without limitations. The present study was designed only to validate the clinical correlation between our objective scale and EOR. Future studies will aim to correlate intraoperative consistency scores to preoperative MRI findings in order to predict tumor consistency in the preoperative planning stage. Future studies will also utilize volumetric EOR to test the association of the meningioma consistency score, as well as distinguishing its relationship to other tumor factors, such as venous sinus invasion and gross adherence to brain, cranial nerves, or vessels. Our study only included meningiomas resected by an open craniotomy. Future studies will aim to assess how consistency score may influence minimally invasive keyhole craniotomies and endoscopic endonasal approaches. Lastly, we plan to design future studies to better assess the genetic and immunohistochemical makeup of tumors with varying consistencies.

\section{Conclusions}

In addition to other important meningioma characteristics such as invasiveness, our study shows that tumor consistency is a key determinant of surgical outcomes including operative duration, complication rates, and EOR, irrespective of tumor location or size. Our grading system also allows surgeons to anticipate the surgical tools and techniques that would be required to achieve maximal EOR. Future studies predicting tumor consistency based on preoperative neuroimaging will help considerably with preoperative planning for meningiomas.

\section{References}

1. Chen TC, Zee CS, Miller CA, Weiss MH, Tang G, Chin L, et al: Magnetic resonance imaging and pathological correlates of meningiomas. Neurosurgery 31:1015-1022, 1992

2. Hoover JM, Morris JM, Meyer FB: Use of preoperative magnetic resonance imaging $\mathrm{T} 1$ and $\mathrm{T} 2$ sequences to determine intraoperative meningioma consistency. Surg Neurol Int 2:142, 2011

3. Hughes JD, Fattahi N, Van Gompel J, Arani A, Meyer F, 
Lanzino G, et al: Higher-resolution magnetic resonance elastography in meningiomas to determine intratumoral consistency. Neurosurgery 77:653-659, 2015

4. Igressa A, Pechlivanis I, Weber F, Mahvash M, Ayyad A, Boutarbouch M, et al: Endoscope-assisted keyhole surgery via an eyebrow incision for removal of large meningiomas of the anterior and middle cranial fossa. Clin Neurol Neurosurg 129:27-33, 2015

5. Khan OH, Anand VK, Schwartz TH: Endoscopic endonasal resection of skull base meningiomas: the significance of a "cortical cuff" and brain edema compared with careful case selection and surgical experience in predicting morbidity and extent of resection. Neurosurg Focus 37(4):E7, 2014

6. Little KM, Friedman AH, Sampson JH, Wanibuchi M, Fukushima T: Surgical management of petroclival meningiomas: defining resection goals based on risk of neurological morbidity and tumor recurrence rates in 137 patients. Neurosurgery 56:546-559, 2005

7. Mawrin C, Perry A: Pathological classification and molecular genetics of meningiomas. J Neurooncol 99:379-391, 2010

8. Murphy MC, Huston J III, Glaser KJ, Manduca A, Meyer FB, Lanzino G, et al: Preoperative assessment of meningioma stiffness using magnetic resonance elastography. J Neurosurg 118:643-648, 2013

9. Muthupillai R, Lomas DJ, Rossman PJ, Greenleaf JF, Manduca A, Ehman RL: Magnetic resonance elastography by direct visualization of propagating acoustic strain waves. Science 269:1854-1857, 1995

10. Ortega-Porcayo LA, Ballesteros-Zebadúa P, MarrufoMeléndez OR, Ramírez-Andrade JJ, Barges-Coll J, Tecante A, et al: Prediction of mechanical properties and subjective consistency of meningiomas using T1-T2 assessment versus fractional anisotropy. World Neurosurg 84:1691-1698, 2015

11. Rehder R, Cohen AR: Endoscope-assisted microsurgical subtemporal keyhole approach to the posterolateral suprasellar region and basal cisterns. World Neurosurg 103:114121,2017

12. Rogers L, Barani I, Chamberlain M, Kaley TJ, McDermott M, Raizer J, et al: Meningiomas: knowledge base, treatment outcomes, and uncertainties. A RANO review. J Neurosurg 122:4-23, 2015

13. Romani R, Tang WJ, Mao Y, Wang DJ, Tang HL, Zhu FP, et al: Diffusion tensor magnetic resonance imaging for predicting the consistency of intracranial meningiomas. Acta Neurochir (Wien) 156:1837-1845, 2014

14. Shiroishi MS, Cen SY, Tamrazi B, D'Amore F, Lerner A, King KS, et al: Predicting meningioma consistency on preoperative neuroimaging studies. Neurosurg Clin N Am 27:145-154, 2016

15. Sitthinamsuwan B, Khampalikit I, Nunta-aree S, Srirabheebhat P, Witthiwej T, Nitising A: Predictors of meningioma consistency: a study in 243 consecutive cases. Acta Neurochir (Wien) 154:1383-1389, 2012

16. Stafford SL, Perry A, Suman VJ, Meyer FB, Scheithauer BW,
Lohse CM, et al: Primarily resected meningiomas: outcome and prognostic factors in 581 Mayo Clinic patients, 1978 through 1988. Mayo Clin Proc 73:936-942, 1998

17. Suzuki Y, Sugimoto T, Shibuya M, Sugita K, Patel SJ: Meningiomas: correlation between MRI characteristics and operative findings including consistency. Acta Neurochir (Wien) 129:39-46, 1994

18. Watanabe K, Kakeda S, Yamamoto J, Ide S, Ohnari N, Nishizawa S, et al: Prediction of hard meningiomas: quantitative evaluation based on the magnetic resonance signal intensity. Acta Radiol 57:333-340, 2016

19. Xu L, Lin Y, Han JC, Xi ZN, Shen H, Gao PY: Magnetic resonance elastography of brain tumors: preliminary results. Acta Radiol 48:327-330, 2007

20. Yamaguchi N, Kawase T, Sagoh M, Ohira T, Shiga H, Toya $\mathrm{S}$ : Prediction of consistency of meningiomas with preoperative magnetic resonance imaging. Surg Neurol 48:579-583, 1997

21. Yamamoto J, Kakeda S, Shimajiri S, Takahashi M, Watanabe K, Kai Y, et al: Tumor consistency of pituitary macroadenomas: predictive analysis on the basis of imaging features with contrast-enhanced 3D FIESTA at 3T. AJNR Am J Neuroradiol 35:297-303, 2014

22. Yano S, Hide T, Shinojima N, Hasegawa Y, Kawano T, Kuratsu J: Endoscopic endonasal skull base approach for parasellar lesions: initial experiences, results, efficacy, and complications. Surg Neurol Int 5:51, 2014

23. Zada G, Yashar P, Robison A, Winer J, Khalessi A, Mack WJ, et al: A proposed grading system for standardizing tumor consistency of intracranial meningiomas. Neurosurg Focus 35(6):E1, 2013

\section{Disclosures}

The authors report no conflict of interest concerning the materials or methods used in this study or the findings specified in this paper.

\section{Author Contributions}

Conception and design: Zada. Acquisition of data: Chang, Itamura, Zada. Analysis and interpretation of data: Chang, Itamura, Donoho, Zada. Drafting the article: Chang, Itamura. Critically revising the article: Chang, Itamura, Donoho, Lucas, Zada. Reviewed submitted version of manuscript: all authors. Approved the final version of the manuscript on behalf of all authors: Chang. Statistical analysis: Chang, Itamura. Administrative/ technical/material support: Giannotta, Zada. Study supervision: Giannotta, Zada.

\section{Correspondence}

Ki-Eun Chang: University of Southern California, Los Angeles, CA. ki.chang14@gmail.com. 